\title{
Effect of different methods of hypoxic exercise training on free radical oxidation and antioxidant enzyme activity in the rat brain
}

\author{
$\mathrm{JIE} \mathrm{LI}^{1}$ and YUXIA WANG ${ }^{2}$ \\ ${ }^{1}$ College of Physical Education, Northwest Normal University, Lanzhou, Gansu 730070; \\ ${ }^{2}$ Physical Education Institute of Nanchang Hangkong University, Nanchang, Jiangxi 330063, P.R. China
}

Received May 23, 2013; Accepted August 12, 2013

DOI: $10.3892 /$ br.2013.162

\begin{abstract}
The effects of different modes of hypoxic exercise training on free radical production and antioxidant enzyme activity in the brain of rats were investigated in this study. A total of 40 healthy 2-month-old male Wister rats were randomly assigned to 5 groups according to different training modes. Endurance training sessions were performed for 5 weeks under different normoxic (atmospheric pressure $\sim 632 \mathrm{mmHg}$, altitude $\sim 1,500 \mathrm{~m}$ ) and hypoxic conditions (atmospheric pressure $\sim 493 \mathrm{mmHg}$, altitude $\sim 3,500 \mathrm{~m}$ ) at the same relative intensity. The superoxide dismutase (SOD), glutathione peroxidase (GSH-Px) and catalase (CAT) activity and the malondialdehyde (MDA) content of the brain were evaluated by spectrophotometric analysis. Compared to the low-training low (LL) group, the SOD activity was significantly increased by $68.73,54.28$ and $304.02 \%$ in the high-training high $(\mathrm{HH})$, high-training low (HL) and high-exercise high-training low (HHL) groups, respectively. However, no obvious change was observed for the low-training high (LH) group. In comparison to the LL group, the GSH-Px activity was found to be significantly higher in the HH, HL, LH and HHL groups. Similarly, in comparison to the LL group, the CAT activity exhibited a significant increase in the HH, HL, LH and HHL groups. Compared to the LL group, the MDA content was significantly increased in the HH, HL and HHL groups, although no significant difference was detected for the LH group. Following exhaustive exercise, the antioxidant enzyme activities in the rat brains were immediately improved in all the hypoxia modes. Moreover, the free radical production was increased after all
\end{abstract}

Correspondence to: Dr Jie Li, College of Physical Education, Northwest Normal University, 967 Anning East Road, Lanzhou, Gansu 730070, P.R. China

E-mail: 1ijie2005ty@163.com

Key words: hypoxic exercise training, brain tissue, malondialdehyde, enzyme activities the modes of hypoxic exercise training, with the LH mode being the only exception.

\section{Introduction}

The human brain constitutes $\sim 2-3 \%$ of the body weight, whereas the blood supply of the brain accounts for $15-20 \%$ of the cardiac output. The cerebral metabolic rate is 7.5 -fold higher compared to the average metabolic rate. The brain readily suffers oxidative damage due to its higher metabolic rate and lipid content and the lower levels of catalase (CAT) and glutathione peroxidase (GSH-Px).

Previous studies indicated that exercise-induced oxidative stress may alter the capacity of oxidation and anti-oxidation of brain tissue (1-3). However, available information regarding the adaptations that occur in the brain tissue during endurance training under hypoxic conditions is limited. The main purpose of this study was to determine whether the activities of antioxidant enzymes, such as superoxide dismutase (SOD), GSH-Px and CAT, were affected in different models of hypoxic exercise training when compared to normoxia. The effects of hypoxic training on lipid peroxidation products, such as malondialdehyde (MDA), were also investigated.

\section{Materials and methods}

Animal training. A total of 40 male specific pathogen-free Wister rats $(\sim 0.13 \mathrm{~kg})$ were obtained from the Animal Science Department, Medical College, Lanzhou University (Lanzhou, China). The rats were housed in barrier facilities in a BioClean room unit and were provided with standard laboratory diet and tap water. The room was maintained at $21-25^{\circ} \mathrm{C}$ and $40-60 \%$ relative humidity. All animal care and procedures were approved by the Institutional Animal Care and Use Committee and the rats were used in accordance with the ethical guidelines of the Northwest Normal University.

To ensure physical exhaustion with the treadmill locomotion, the rats were accustomed to running on a Quinton rodent treadmill (Quinton Instruments, Seattle, WA, USA) for $15 \mathrm{~min}$ per day, with the speed being gradually increased from 12 to $35 \mathrm{~m} / \mathrm{min}$ within 1 week. The living condition was set in the hypoxic cabin ( $2 \mathrm{~h}$ /day) for 2 days and the designed altitude 
Table I. Training modes in normoxia.

\begin{tabular}{ccccc}
\hline Week & $\begin{array}{c}\text { Speed } \\
(\mathrm{m} / \mathrm{min})\end{array}$ & $\begin{array}{c}\text { Gradient } \\
\left(^{\circ}\right)\end{array}$ & $\begin{array}{c}\text { Training time } \\
(\mathrm{min} / \text { day })\end{array}$ & $\begin{array}{c}\text { Training days } \\
(\text { per week })\end{array}$ \\
\hline 1 & 25 & 0 & 30 & 6 \\
2 & 30 & 0 & 40 & 6 \\
3 & 30 & 0 & 50 & 6 \\
4 & 30 & 0 & 60 & 6 \\
5 & 35 & 0 & 60 & 6 \\
\hline
\end{tabular}

Table II. Training modes in hypoxia.

\begin{tabular}{ccccc}
\hline Week & $\begin{array}{c}\text { Speed } \\
(\mathrm{m} / \mathrm{min})\end{array}$ & $\begin{array}{c}\text { Gradient } \\
\left({ }^{\circ}\right)\end{array}$ & $\begin{array}{c}\text { Training time } \\
(\mathrm{min} / \text { day })\end{array}$ & $\begin{array}{c}\text { Training days } \\
\text { (per week) }\end{array}$ \\
\hline 1 & 20 & 0 & 30 & 6 \\
2 & 25 & 0 & 40 & 6 \\
3 & 25 & 0 & 50 & 6 \\
4 & 25 & 0 & 60 & 6 \\
5 & 30 & 0 & 60 & 6 \\
\hline
\end{tabular}

was gradually increased from 2,500 to $3,500 \mathrm{~m}$. The rats were subsequently running on the treadmill (DSPT-202; Qianjiang Industry and Trade Co., Ltd., Hangzhou, China) for 4 days under hypoxic conditions at the designed altitude of $3,500 \mathrm{~m}$, with the speed being gradually increased from 20 to $30 \mathrm{~m} / \mathrm{min}$ for a time period of 30-60 min. The training mode was modified from (4): training under normoxic conditions at the altitude of $\sim 1,500 \mathrm{~m}$ (Table I) and under hypoxic conditions at the altitude of 3,500 $\mathrm{m}$ (Table II).

Exercise protocol. The rats were randomly assigned to the following 5 groups according to the different training modes: low-training low (LL group, $n=8$ ), trained in normoxia and kept in normoxia for $24 \mathrm{~h}$ on non-training days (5); high-training high (HH group, $\mathrm{n}=8$ ), trained in hypoxia and kept in hypoxia for $24 \mathrm{~h}$ on non-training days (6); high-training low (HL group, $\mathrm{n}=8$ ), kept in hypoxia for $12 \mathrm{~h}$ and trained in normoxia for the remaining $12 \mathrm{~h}$, with $12 \mathrm{~h}$ in hypoxia and $12 \mathrm{~h}$ in normoxia on each non-training day; low-training high (LH group, $\mathrm{n}=8$ ), trained in hypoxia with the altitude of $\sim 3,500 \mathrm{~m}$ and kept in normoxia for $24 \mathrm{~h}$, with $24 \mathrm{~h}$ in normoxia on non-training days; and high-exercise high-training low (HHL group, $\mathrm{n}=8$ ), with a similar exercise protocol to the HL group and the addition of a hypoxic training every other day on training days.

Tissue preparation. Following a 5-week exercise training, the rats were transferred to a normoxic environment for 3 days without any training. Subsequently, an acute bout of treadmill running at $35 \mathrm{~m} / \mathrm{min}$ and $0 \%$ grade until exhaustion in normoxia was conducted as previously described $(7,8)$. All the rats were sacrificed by decollation immediately after the exercise. The brain was excised, frozen in liquid nitrogen and stored at $-20^{\circ} \mathrm{C}$ for further assessment. A brain tissue sample was thawed in a buffer containing $70 \mathrm{mM}$ sucrose, $10 \mathrm{mM}$ Tris- $\mathrm{HCl}, 0.5 \mathrm{mM}$ EDTA and $210 \mathrm{mM}$ mannitol, supplemented with $0.2 \%$ bovine serum albumin ( $\mathrm{pH} 7.4,1: 10 \mathrm{w} / \mathrm{v})$ and homogenized with YQ-3 dynamoelectric homogenizer (Jintan, Jiangsu, China) at $0^{\circ} \mathrm{C}$. Following centrifugation at $14,255 \mathrm{x}$ g for $15 \mathrm{~min}$ at $0^{\circ} \mathrm{C}$, the supernatant was collected for analysis.

Biochemical analyses. All the spectrophotometric assays were performed with an UV-754N ultraviolet scanning spectrophotometer (Shanghai Exactitude Scientific Apparatus Ltd., Shanghai, China).

Antioxidant enzyme activities. The SOD activity was determined at $25^{\circ} \mathrm{C}$ by autoxidation of pyrogallic acid and one enzyme unit was defined as the amount of enzyme required to cause a 50\% inhibition of pyrogallol autoxidation (9). The GSH-Px activity was assayed at $37^{\circ} \mathrm{C}$ as previously described by Xu et al (9). The CAT activity was determined at $20^{\circ} \mathrm{C}$ as described by Stellmach (10).

Lipid peroxidation. Brain lipid peroxidation was detected by measuring MDA (Jiancheng Bioengineering Research Institute, Nanjing, China) in thiobarbituric acid (TBA) (Sigma, St. Louis, MO, USA), according to the procedure previously described by $\mathrm{Li}(11)$.

Protein concentration. The protein concentration determination was conducted using a Coomassie Brilliant Blue kit (Sigma) with bovine serum albumin (12).

Statistical analysis. SPSS software, version 13.0 (SPSS Inc., Chicago, IL, USA) was used for Student's unpaired t-test statistical analysis. Data are expressed as means \pm standard deviation. One-way analysis of variance (ANOVA) was applied to compare the differences among the different groups. $\mathrm{P}<0.05$ was considered to indicate a statistically significant difference.

\section{Results}

Body weight. To determine the effects of compound hypoxic training on body weight, data were collected from the training groups and the normal-treated group. The pre-training and post-training body weights ( $\mathrm{W}_{0}$ and $\mathrm{W}_{5}$, respectively) are shown in Table III. Prior to exercise training $\left(\mathrm{W}_{0}\right)$, there were no body weight differences between each of the hypoxic groups (HH, HL, LH and HHL) and the normoxic group (LL). At the end of the training $\left(\mathrm{W}_{5}\right)$, although the body weight of all the rats was increased, no significant difference was observed between any of the hypoxic groups and the normoxic group. These results suggested that the hypoxic modes exerted no effect on weight change.

Endurance time. To access the athletic performance in the training groups, exhaustive exercise was performed. After a 5 -week training, the endurance times of running to exhaustion are summarized in Table IV. The time in the HHL group was longer compared to that in the LL group $(\mathrm{P}=0.040)$. However, there were no significant differences between the LL group and any of the other three hypoxic groups. These results suggested that the improvement in the athletic performance in the hypoxic 
Table III. Body weight.

\begin{tabular}{lccccr}
\hline Training mode & LL & HH & HL & LH & HHL \\
\hline Pre-training $\left(\mathrm{W}_{0}\right)$ & $127.2 \pm 22.8$ & $131.9 \pm 7.8$ & $141.5 \pm 12.3$ & $129.6 \pm 14.6$ & $138.3 \pm 10.8$ \\
Post-training $\left(\mathrm{W}_{5}\right)$ & $210.6 \pm 26.5$ & $227.5 \pm 28.3$ & $208.0 \pm 20.1$ & $226.8 \pm 28.8$ & $205.8 \pm 24.0$
\end{tabular}

Weight $(\mathrm{g})$ is expressed as means $\pm \mathrm{SD}$ for 8 rats per group. $\mathrm{LL}$, living low-training low; $\mathrm{HH}$, living high-training high; HL, living high-training low; LH, living low-training high; HHL, living high-exercise high-training low. SD, standard deviation.

Table IV. Endurance time.

\begin{tabular}{lccccc}
\hline Training mode & LL & HH & HL & LH & HHL \\
\hline Endurance time & $187.63 \pm 52.81$ & $219.38 \pm 29.05$ & $178.25 \pm 36.85$ & $169.00 \pm 28.52$ & $224.88 \pm 17.62^{\mathrm{a}}$ \\
\hline
\end{tabular}

${ }^{\mathrm{a}} \mathrm{P}<0.05$. Time $(\mathrm{min})$ is expressed as means $\pm \mathrm{SD}$ for 8 rats per group. LL, living low-training low; HH, living high-training high; HL, living high-training low; LH, living low-training high; HHL, living high-exercise high-training low. SD, standard deviation.

Table V. Antioxidant enzyme activities.

\begin{tabular}{lccccc}
\hline Training mode & LL & HH & HL & LH & HHL \\
\hline SOD & $25.960 \pm 10.227$ & $69.669 \pm 27.610^{\mathrm{b}}$ & $66.010 \pm 29.939^{\mathrm{a}}$ & $22.668 \pm 9.159$ & $130.843 \pm 19.139^{\mathrm{b}}$ \\
GSH-Px & $15.855 \pm 5.993$ & $310.574 \pm 46.703^{\mathrm{b}}$ & $90.144 \pm 49.829^{\mathrm{a}}$ & $82.325 \pm 50.960^{\mathrm{a}}$ & $106.833 \pm 38.869^{\mathrm{b}}$ \\
CAT & $22.307 \pm 3.947$ & $34.563 \pm 3.944^{\mathrm{b}}$ & $30.507 \pm 4.128^{\mathrm{b}}$ & $31.132 \pm 3.573^{\mathrm{b}}$ & $38.704 \pm 4.647^{\mathrm{b}}$ \\
\hline
\end{tabular}

${ }^{a} \mathrm{P}<0.05$ and ${ }^{\mathrm{b}} \mathrm{P}<0.001$ compared to the LL group. Enzyme activities (U/mg protein) are expressed as means \pm SD for 8 rats per group. LL, living low-training low; HH, living high-training high; HL, living high-training low; LH, living low-training high; HHL, living high-exercise high-training low; SOD, superoxide dismutase; GSH-Px, glutathione peroxidase; CAT, catalase. SD, standard deviation.

Table VI. Malondialdehyde content.

\begin{tabular}{|c|c|c|c|c|c|}
\hline Training mode & LL & $\mathrm{HH}$ & HL & LH & HHL \\
\hline MDA & $0.286 \pm 0.099$ & $0.843 \pm 0.121^{\mathrm{b}}$ & $0.386 \pm 0.088^{a}$ & $0.302 \pm 0.088$ & $0.500 \pm 0.050^{\mathrm{b}}$ \\
\hline
\end{tabular}

training model was not significant compared to the normal training model.

Antioxidant enzyme activity. To determine the effect of hypoxic training on the activity of antioxidant enzymes, the activity of SOD, GSH-Px and CAT was assessed (Table V). Compared to the LL group, the SOD activity was significantly increased immediately after running to exhaustion in the $\mathrm{HH}$, HL and HHL hypoxic groups. However, no obvious differences were observed between the LH and LL groups. The GSH-Px activity in the four hypoxic groups was significantly higher compared to that in the LL group. CAT exhibited a similar activity pattern to GSH-Px, showing a statistically significant increase in groups HH, HL, LH and HHL, compared to the LL group. These results suggested that the activity of SOD, GSH-Px and CAT may be significantly increased with hypoxic training, with the LH model being the only exception.

MDA content. As an index of tissue lipid peroxidation under peroxide- or exercise-induced oxidative stress, the MDA content was measured and shown in Table VI. The results indicated that the MDA level in groups HH, LH and HHL was obviously higher compared to that in the LL group. However, no significant differences were observed between the LH and LL groups. 
These results suggested that only the HH and HHL training models may increase the MDA content to a significant level.

\section{Discussion}

The data presented in this study demonstrated that the rat weight in all the groups was increased after 5 weeks of feeding and training. However, there was no significant difference aamong the training modes. Of note, compared to the normoxic group, the increase of the rat weights in the hypoxic groups exhibited a consistent tendency, suggesting that neither the hypoxic nor the normoxic training affected rat weight.

Endurance time is widely accepted as an index to measure sports ability. In the present study, the endurance time in the HHL group was significantly longer $(\mathrm{P}<0.05)$, whereas the remaining three modes of hypoxic treatment $(\mathrm{HH}, \mathrm{HL}$ and $\mathrm{LH}$ ) exhibited no significant difference in endurance time compared to the normoxic group (LL). The stimulation mechanism of the exercise training mode in hypoxia requires further investigation.

Due to the high content of unsaturated fatty acids and the free radical metabolism, lipid peroxidation is active in brain tissue. It was previously demonstrated that, compared to the control (no training treatment), the SOD activity in the rat brain was significantly increased in the common-loads group (running on the treadmill for 3 and 7 weeks), whereas no significant difference was observed in the extreme-loads group (running on the treadmill for 3, 5 and 7 weeks with ascending speed) (13). Of note, compared to the common-loads group, a significant decrease in the SOD activity was observed in the extreme-loads group, which may be attributed to the exercise-induced fatigue (13). Jiang and Lei (14) reported that intermittent hypoxic exposure for 4 weeks may significantly increase the SOD activity and reduce the MDA content of the rat brain. Moreover, the combined treatment of intermittent hypoxic exposure with swimming exercise led to a sharp increase in the SOD activity and a marginal decrease in the MDA content (14). Therefore, appropriate exercise with hypoxic exposure provides an effective approach to improving the antioxidant capacity of the rat brain (14).

In this study, following exhaustive running for 5 weeks, the SOD activity in the HH, HL and HHL hypoxic groups was significantly higher compared to that in the normoxic LL group, with the increase being 168.371, 154.276 and $404.018 \%$, respectively. However, there was no significant change in the SOD activity in the LH group, which may be explained by the lack of hypoxic time to stimulate the functional adaptations.

Similarly, the GSH-Px activity in the brain of rats from the hypoxic exercise training groups HH, HL LH and HHL, was increased by $1,858.39,468.553,419.237$ and $573.813 \%$, respectively. These statistical data suggest that the combined mode of exercise training and hypoxic stimulation was more efficient in improving brain GSH-Px activity compared to exercise training alone and the noticeable sharp increase during HH training may be attributed to further hypoxic stimulation.

Compared to the normoxic LL group, the CAT activity was significantly increased in the four hypoxic exercise training groups, exhibiting a similar increase pattern to that of SOD and GSH-Px. The relative increase in the HH (54.942\%), HL
(36.760\%), LH (39.562\%) and HHL (73.506\%) groups indicated that exercise training combined with hypoxic stimulation was more efficient in improving brain CAT activity compared to exercise training alone.

As the result of free radicals attacking the membrane components of the cell, lipid peroxidation is frequently used as an indicator of tissue oxidation $(15,16)$. MDA accumulation following strenuous exercise has been widely reported in the skeletal muscle, liver and plasma (17-19). A previous study (13) also demonstrated that MDA was significantly increased following a 3-week exercise training and extreme loads. In this study, the MDA content in the brain of exhaustively exercised rats from the hypoxic groups $\mathrm{HH}, \mathrm{HL}$ and HHL was higher compared to the normoxic LL group, with the increase being $194.755,34.965$ and $74.825 \%$, respectively. These statistical results indicate that exercise training combined with hypoxia led to a high content of MDA in brain. However, there was no significant difference between the LH and LL groups, which may be attributed to the lack of time for hypoxic stimulation.

Generally, the combined mode of hypoxia with exercise training may increase the antioxidant enzyme activity in the rat brain immediately following an acute bout of exhaustive exercise. However, the exercise-induced brain oxidative damage was found to be more severe due to the hypoxic environment and the resulting exercise-induced free radical production. As regards exhaustive exercise, the training mode in hypoxia with an altitude of $\sim 3,500 \mathrm{~m}$ and being kept in normoxia for $24 \mathrm{~h}$, with $24 \mathrm{~h}$ in normoxia on non-training days, was shown to improve the antioxidant enzyme activity in the rat brain.

\section{Acknowledgements}

This study was supported by the National Natural Science Foundation of China (no. 31060145) and the Natural Science Foundation of Gansu province (no. 1107RJZA087).

\section{References}

1. Cao GH and Chen JL: The effect of swimming on the free radical metabolism in brain tissue of rats. Chin J Sports Med 9: 149-151, 1990 (In Chinese).

2. Xin D, Li H, Li JX, et al: Dynamic observation of production of free radical and oxidative, antioxidative capability of brain in rats during exhausting exercise. Chin J Sports Med 18: 321-323, 1999 (In Chinese).

3. Zhang YK, Jiao Y, Zheng SQ, et al: The effects of exhaustive swimming on the free radical metabolism and serum CK, LDH activity in rats. Chin J Sports Med 12: 69-72, 1995 (In Chinese).

4. Lu Z, Feng LS, Zhao P, et al: Effect of different modes of hypoxia training on the capability of rat blood carrying oxygen. Chin J Sports Med 26: 68-70, 2007 (In Chinese).

5. Zheng L and Lu AY: The research on animal model of exercise fatigue. China Sport Sci Tech 39: 20-23, 2003 (In Chinese).

6. Bigard AX, Brunet A, Guezennec CY and Monod H: Skeletal muscle changes after endurance training at high altitude. J Appl Physiol 71: 2114-2121, 1991.

7. Tian Y and Gao TQ: The establishment of exercise-induced fatigue models in rats. J Beijing Univ Phys Educ 18: 49-53, 1995 (In Chinese).

8. Ji LL and Fu RG: Responses of glutathione system and antioxidant enzymes to exhaustive exercise and hydroperoxide. J Appl Physiol 72: 549-554, 1992.

9. Xu SY, Bian RL and Chen X (eds): Methodology of Pharmacological Experiments. 3rd edition. People's Medical Publishing House, Beijing, 2002.

10. Stellmach B (ed): Bestimmungsmethoden Enzyme. China Light Industry Press, pp186-188, 1992. 
11. Li YK (ed): Experimental Methodology of Traditional Chinese Medicine Pharmacology. Shanghai Scientific and Technical Publishers, Shanghai, p202, 1991.

12. Yuan HQ: Experiment of Medical Molecular Cell Biology. Science Press 8, 2007.

13. You CY, Zuo HW, Tian YP and Shen WM: Effect of different loads treadmill-running on free radical metabolism and activities of antioxidant enzyme system. Chin J Sports Med 20: 202-204, 2001 (In Chinese).

14. Jiang ML and Lei ZP: Effects of free radical metabolic on interval hypoxia exposure of mice. Chin J Sports Med 24: 87-88, 2005 (In Chinese).

15. Halliwell B and Gutteridge JMC (eds): Free Radicals in Biology and Medicine. 1st edition. Clarendon Press, Oxford, pp73-75, 104-106, 1985.
16. Slater TF, Cheeseman KH, Davies MJ, Proudfoot K and Xin W: Free radical mechanisms in relation to tissue injury. Proc Nutr Soc 46: 1-12, 1987

17. Davies KJ, Quintanilha AT, Brooks GA and Packer L: Free radicals and tissue damage produced by exercise. Biochem Biophys Res Commun 107: 1198-1205, 1982.

18. Duthie GG, Robertson JD, Maughan RJ and Morrice PC: Blood antioxidant status and erythrocyte lipid peroxidation following distance running. Arch Biochem Biophys 282: 78-83, 1990.

19. Kanter MM, Hamlin RL, Unverferth DV, Davis HW and Merola AJ: Effect of exercise training on antioxidant enzymes and cardiotoxicity of doxorubicin. J Appl Physiol 59: 1298-1303, 1985. 